\title{
A Modified Semi-Analytic Iterative Method for Solving a Class of Partial Integro-Differential Equations with Conformable Fractional Order Derivative
}

\author{
Ahmed Salim*, Osama H. Mohammed \\ Department of Mathematics, College of Science, Al-Nahrain University, Baghdad, Iraq
}

\begin{tabular}{lr}
\multicolumn{1}{c}{ Articles Information } & Abstract \\
\hline Received: & \\
29.01 .2020 & \\
Accepted: & \\
20.05 .2020 & In this paper, we introduce a modified semi analytic iterative \\
Published: & method for solving conformable fractional Partial Integro- \\
04.06 .2020 & Differential equations (CFPIDEs). The methodology is tested by \\
\hline & some illustrative examples which are given to demonstrate its \\
Keywords: & accuracy, applicability and efficiency. \\
Partial Integro-Differential equations (PIDEs) & \\
Conformable fractional derivative & \\
Shifted Legendre polynomials (SLPs) & \\
& \\
\hline
\end{tabular}

DOI: $10.22401 /$ ANJS.23.2.07

*Corresponding author: ahmedsalim.5993@gmail.com

\section{Introduction}

Fractional calculus is a very strong tool and it is considered as one of the best ways to express the physical models and engineering processes. The part fractional calculus takes in many different fields such as mechanics, chemistry, economics is very significant and important [1-3]. Integro-differential equations play a fundamental role in the studies of linear and nonlinear functional analysis. There are several definitions for the fractional derivatives but recently, the authors in [4] gave a new uncomplicated definition based on the usual derivative definition which is called conformable fractional derivative. Fractional partial integro-differential equations (FPIDEs) occur naturally in many different fields of science, engineering and social sciences. Applications of FPIDEs can be found in many fields such as [5-12]. This paper is concerned with obtaining approximate solutions of the following CFPIDE: subject to

$$
T_{y}^{v} \gamma(y, t)=g(y, t)+I_{t}^{v} I_{y}^{v} \gamma(y, t)
$$

$$
\gamma(0, t)=b(t)
$$

where $y$ and $t$ are the independent variables, $g(y, t)$ is a known function, $\gamma \in C_{y}([0,1] \times[0,1])$ is an unknown function to be evaluated, $T_{y}^{v}$ is the conformable fractional derivative and $I_{t}^{v}, I_{y}^{v}$ are the conformable fractional integral of order $v \in\left(\frac{1}{2}, 1\right) /\left(\frac{7}{10}, \frac{75}{10}\right)$ with respect to $t$ and $y$, respectively. The paper is organized as follows: in section 2, some of the basic concepts of the conformable fractional order derivative are presented. In section 3, we introduce the shifted Legendre polynomials. While in section 4 , existence of a unique solution for problem (1)-(2) is proved. The proposed method for solving problem (1)-(2) is introduced in section 5. In section 6, the accuracy of the proposed technique is checked by solving some numerical examples. Finally, a conclusion has been given.

\section{Preliminaries:}

This section presents the definition of the Conformable fractional derivative and some of its properties that has been used in this article.

Definition (1), [4]: The conformable fractional order derivative of order $\mathrm{v}$ of a function $\gamma:[0, \infty) \rightarrow \mathbb{R}$ is given by:

$$
T_{t}^{v}(\gamma)(t)=\lim _{\varepsilon \rightarrow 0} \frac{\gamma\left(t+\varepsilon t^{1-v}\right)-\gamma(t)}{\varepsilon}
$$

for all $t>0, v \in(0,1)$. If $\gamma$ is $\mathrm{v}$-differentiable in some $(0, a), a>0$, and $\lim _{t \rightarrow 0^{+}} \gamma^{(v)}(t)$ exists, then define

$$
\gamma^{(v)}(0)=\lim _{t \rightarrow 0^{+}} \gamma^{(v)}(t) .
$$




\section{Al-Nahrain Journal of Science}

ANJS, Vol.23 (2), June, 2020, pp. $44-51$

Definition (2), [4]:

$$
I_{t}^{v}(\gamma)(t)=I_{1}^{v}\left(t^{v-1} \gamma\right)=\int_{a}^{t} \frac{\gamma(s)}{s^{1-v}} d s,
$$

and the integral here is the usual Riemann integral, and $v \in(0,1), a \geq 0$.

\section{Properties, [4]:}

1. $T_{t}^{v}\left(t^{p}\right)=p t^{p-v}, \quad \forall p \in \mathbb{R}$.

2. If, in addition, $f$ is differentiable, then $T_{t}^{v}(f)(t)=$ $t^{1-v} \frac{d f}{d t}(t)$

3. $T^{v}(a f+b g)=a T^{v}(f)+b T^{v}(g), \forall a, b \in \mathbb{R}$.

4. $T^{v}(\lambda)=0$, for any constant function $f(\tau)=\lambda$.

5. $T^{v}(f g)=f\left(T^{v}(g)\right)+g\left(T^{v}(f)\right)$.

6. $T^{v}\left(\frac{f}{g}\right)=\frac{g T^{v}(f)-f T^{v}(g)}{g^{2}}, g \neq 0$.

Theorem (1), [4]: Let $f:(a, b) \rightarrow \mathbb{R}, v \in(0,1)$ if $\mathrm{f}$ is a differentiable function. Then for all $\tau>$ a we have

$$
I_{\alpha}^{a} T_{\alpha}^{a}(f)(\tau)=f(t)-f(a) .
$$

Theorem (2), [13]: $T_{\tau}^{v} I_{\tau}^{v}(f)(\tau)=f(\tau)$, for $\tau \geq 0$, where $f$ is any continuous function in the domain of $I_{\tau}^{v}$.

\section{Shifted Legendre Polynomials (SLP)}

The Legendre polynomial of degree $j$, denoted by $L_{j}(\tau)$, defined on the interval $[-1,1]$, can be generated as follows [14]:

$$
L_{j+1}(\tau)=\frac{(2 j+1)}{(j+1)} \tau L_{j}(\tau)-\frac{j}{j+1} L_{j-1}(\tau), \quad j=1,2, \ldots .
$$

On the other hand, the SLPs, denoted by $\mathcal{L}_{l, j}(t)$ defined on $[0, l]$, can be introduced if we assume $\tau=\frac{2 t}{l}-1$, then the polynomials are constructed using the following formula,

$$
\begin{gathered}
\mathcal{L}_{l, j+1}(t)=\frac{(2 j+1)}{(j+1)}\left(\frac{2 t}{\imath}-1\right) \mathcal{L}_{l, j}(t)- \\
\frac{j}{j+1} \mathcal{L}_{l, j-1}(t), \quad j=1,2, \ldots \\
\mathcal{L}_{l, 0}(t)=1 \text { and } \mathcal{L}_{l, 1}(t)=\frac{2 t}{\imath}-1 . \iota \neq 0
\end{gathered}
$$

The orthogonality relation can be introduced as $\int_{0}^{1} \mathcal{L}_{l, i}(t) \mathcal{L}_{l, j}(t) d t= \begin{cases}\frac{l}{2 i+1}, & i=j \\ 0, & i \neq j .\end{cases}$

A function $\gamma(t) \in L_{2}(0, \imath)$, can be decomposed regarding the SLPs as:

$$
\gamma(t)=\sum_{j=0}^{\infty} c_{j} \mathcal{L}_{l, j}(t)
$$

where the coefficients $c_{j}$ can take the form

$c_{j}=\frac{(2 j+1)}{l} \int_{0}^{l} \gamma(t) \mathcal{L}_{l, j}(t) d t, \quad j=0,1, \ldots$

If we truncate the summation in equation (5) after $n$ terms, then $\gamma(t)$ will be approximated by $\gamma_{n}(t)$ as:

$$
\gamma_{n}(t) \simeq \sum_{j=0}^{n} c_{j} \mathcal{L}_{l, j}(t),
$$

Remark (1): A function $\gamma(y, t)$ of two independent variables defined for $(y, t) \in[0,1] \times[0,1]$ can be expanded in terms of the SLPs as:

$$
\gamma_{n, m}(x, t) \simeq \sum_{i=0}^{n} \sum_{j=0}^{m} c_{i j} \mathcal{L}_{1, i}(y) \mathcal{L}_{1, j}(t),
$$

where $a_{i j}$ are given by

$$
\begin{gathered}
c_{i j}= \\
(2 i+1)(2 j+1) \int_{0}^{1} \int_{0}^{1} \gamma(y, t) \mathcal{L}_{1, i}(y) \mathcal{L}_{1, j}(t) d y d t, \\
i=0,1, \ldots, n, \quad j=0,1, \ldots, m .
\end{gathered}
$$

\section{Existence and Uniqueness:}

In this section, the existence and uniqueness of problem (1)-(2) will be introduced. The proof of the main result is based on the concept of Banach fixed point theorem.

Definition (3), [15]: Let (B, $\|\|$.$) be a Banach space$ and let $T: \mathrm{B} \rightarrow \mathrm{B}$. Then $T$ is said to be $k$-contraction if there exist a constant $k \in(0,1)$ such that

$$
\|T u-T v\| \leq k\|u-v\|
$$

for all $u, v \in \mathrm{B}$.

Theorem (3), [15]: Let (B, $\|\|$.$) be a Banach space and$ let $T: \mathrm{B} \rightarrow \mathrm{B}$ be a $k$-contraction. Then $\mathrm{T} \gamma=\gamma$ has a unique solution, that is, $\mathrm{T}$ has only one fixed point $\gamma^{*} \in \mathrm{B}$.

Definition (4): Let $\|\cdot\|_{B, \lambda}: X \rightarrow \mathbb{R}^{+}$where

$\|\gamma\|_{B, \lambda}=\max _{(y, t) \in[0,1] \times[0,1]}|\gamma(y, t)| e^{-\lambda y t}$

for some suitable $\lambda>0$.

Lemma (1): The function $\gamma \in X$ is a solution of problem (1)-(2) if and only if $\gamma(y, t)$ satisfies

$$
\gamma(y, t)=b(t)+{ }_{y} I_{v}^{0} g(y, t)+{ }_{y} I_{v}^{0} I_{v}^{0} y I_{v}^{0} \gamma(y, t)
$$

Proof: Apply ${ }_{y} I_{v}^{0}$ on both sides of equation (1), yields ${ }_{y} I_{v}^{0} T_{y}^{v} \gamma(y, t)={ }_{y} I_{v}^{0} g(y, t)+{ }_{y} I_{v}^{0} I_{v}^{0} y_{v}^{0} \gamma(y, t)$.

According to equation (2) and theorem (1), we have 


\section{Al-Nahrain Journal of Science}

ANJS, Vol.23 (2), June, 2020, pp. $44-51$

$$
\gamma(y, t)=b(t)+{ }_{y} I_{v}^{0} g(y, t)+{ }_{y} I_{v}^{0} I_{v y}^{0} I_{v}^{0} \gamma(y, t) .
$$

And hence the result is obtained.

Theorem (4): Let $T:\left(X,\|\cdot\|_{B, \lambda}\right) \rightarrow\left(X,\|\cdot\|_{B, \lambda}\right)$ be defined as

$$
T \gamma(y, t)=b(t)+{ }_{y} I_{v}^{0} g(y, t)+{ }_{y} I_{v}^{0} t_{v}^{0} I_{v}^{0} \gamma(y, t)
$$

if

$$
\begin{aligned}
& \lambda>\left[\frac{1}{(2 v-1)(4 v-3)(10 v-7)}\right]^{\frac{1}{7-8 v}} \text { and } \\
& v \in\left(\frac{1}{2}, 1\right) /\left(\frac{7}{10}, \frac{75}{10}\right)
\end{aligned}
$$

then $T$ has a unique solution.

Proof: Let $\gamma_{1}, \gamma_{2} \in X$, we have

$$
\begin{gathered}
\left|T \gamma_{1}(y, t)-T \gamma_{2}(y, t)\right|=\left|y_{v}^{0} I_{v y}^{0} I_{v}^{0}\left[\gamma_{1}(y, t)-\gamma_{2}(y, t)\right]\right| \\
\leq \int_{0}^{y} \int_{0}^{t} \int_{0}^{z} \frac{\left|\gamma_{1}(u, s)-\gamma_{2}(u, s)\right|}{\left|u^{1-v} \| s^{1-v}\right|\left|z^{1-v}\right|} d u d s d z \\
\leq\left\|\gamma_{1}-\gamma_{2}\right\|_{B, \lambda} \\
\quad \int_{0}^{y} \int_{0}^{t} \int_{0}^{z} \frac{e^{\lambda u s}}{u^{1-v} s^{1-v} z^{1-v}} d u d s d z .
\end{gathered}
$$

Making the change of variables $\mu_{1}=\lambda u s, 0 \leq \mu_{1} \leq \lambda z s$, we get

$\left|T \gamma_{1}(y, t)-T \gamma_{2}(y, t)\right| \leq \frac{1}{\lambda^{v}}\left\|\gamma_{1}-\gamma_{2}\right\|_{B, \lambda}$

$$
\int_{0}^{y} \int_{0}^{t} \frac{1}{s z^{1-v}} \int_{0}^{\lambda z s} \mu_{1}{ }^{v-1} e^{\mu_{1}} d \mu_{1} d s d z
$$

By using Holder's inequality, for $v \in\left(\frac{1}{2}, 1\right) /\left(\frac{7}{10}, \frac{75}{10}\right)$, we have

$\int_{0}^{\lambda z s} \mu_{1}{ }^{v-1} e^{\mu_{1}} d \mu_{1} \leq$

$\left(\int_{0}^{\lambda z s} \mu_{1}^{2(v-1)} d \mu_{1}\right)^{1 / 2}\left(\int_{0}^{\lambda z s} e^{2 \mu_{1}} d \mu_{1}\right)^{1 / 2} \leq \frac{(\lambda z s)^{2 v-1}}{(2 v-1)} e^{\lambda z s}$.

Then

$\left|T \gamma_{1}(y, t)-T \gamma_{2}(y, t)\right| \leq \frac{\lambda^{2 v-1}}{\lambda^{v}(2 v-1)}\left\|\gamma_{1}-\gamma_{2}\right\|_{B, \lambda}$

$$
\int_{0}^{y} \int_{0}^{t} \frac{z^{2 v-1} s^{2 v-1}}{s z^{1-v}} e^{\lambda z s} d s d z
$$

$\left|T \gamma_{1}(y, t)-T \gamma_{2}(y, t)\right| \leq \frac{\lambda^{v-1}}{(2 v-1)}\left\|\gamma_{1}-\gamma_{2}\right\|_{B, \lambda}$

$$
\int_{0}^{y} \int_{0}^{t} z^{3 v-2} s^{2(v-1)} e^{\lambda z s} d s d z .
$$

By change of variable $\mu_{2}=\lambda z s, 0 \leq \mu_{2} \leq \lambda z t$, we get

$$
\left|T \gamma_{1}(y, t)-T \gamma_{2}(y, t)\right| \leq \frac{\lambda^{v-1}}{(2 v-1)}\left\|\gamma_{1}-\gamma_{2}\right\|_{B, \lambda}
$$

$$
\int_{0}^{y} \frac{z^{v-1}}{\lambda^{2 v-1}} \int_{0}^{\lambda z t} \mu_{2}{ }^{2(v-1)} e^{\mu_{2}} d \mu_{2} d z
$$

Using Holder inequality for $v \in\left(\frac{1}{2}, 1\right) /\left(\frac{7}{10}, \frac{75}{10}\right)$, we get

$$
\begin{aligned}
& \int_{0}^{\lambda z t} \mu_{2}{ }^{2(v-1)} e^{\mu_{2}} d \mu_{2} \\
& \leq\left(\int_{0}^{\lambda z t} \mu_{2}{ }^{4(v-1)} d \mu_{2}\right)^{1 / 2}\left(\int_{0}^{\lambda z t} e^{2 \mu_{2}} d \mu_{2}\right)^{1 / 2} \\
& \leq \frac{(\lambda z)^{4 v-3}}{(4 v-3)} e^{\lambda z t} .
\end{aligned}
$$

Then

$\left|T \gamma_{1}(x, t)-T \gamma_{2}(x, t)\right| \leq \frac{\lambda^{v-1} \lambda^{2(v-1)}}{(2 v-1)(4 v-3)}\left\|\gamma_{1}-\gamma_{2}\right\|_{B, \lambda}$

$$
\int_{0}^{y} z^{5 v-4} e^{\lambda z t} d z
$$

And finally using change of variables $\mu_{3}=\lambda z t$,

$0 \leq \mu_{3} \leq \lambda y t$, we get

$\left|T \gamma_{1}(y, t)-T \gamma_{2}(y, t)\right| \leq \frac{\lambda^{v-1} \lambda^{2(v-1)}}{(2 v-1)(4 v-3)}\left\|\gamma_{1}-\gamma_{2}\right\|_{B, \lambda}$

$$
\frac{1}{\lambda^{5 v-3}} \int_{0}^{\lambda y t} \mu_{3}^{5 v-4} e^{\mu_{3}} d \mu_{3}
$$

By using Holder inequality for $v \in\left(\frac{1}{2}, 1\right) /\left(\frac{7}{10}, \frac{75}{10}\right)$, we have

$\int_{0}^{\lambda y t} \mu_{3}{ }^{5 v-4} e^{\mu_{3}} d \mu_{3} \leq$

$\left(\int_{0}^{\lambda y t} \mu_{3}{ }^{2(5 v-4)} d \mu_{3}\right)^{1 / 2}\left(\int_{0}^{\lambda y t} e^{2 \mu_{3}} d \mu_{3}\right)^{1 / 2} \leq \frac{\lambda^{10 v-7}}{(10 v-7)} e^{\lambda y t}$.

\section{Then}

$\left|T \gamma_{1}(y, t)-T \gamma_{2}(y, t)\right|$

$\leq \frac{\lambda^{v-1} \lambda^{2(v-1)} \lambda^{5 v-4}}{(2 v-1)(4 v-3)(10 v-7)} e^{\lambda y t}\left\|\gamma_{1}-\gamma_{2}\right\|_{B, \lambda}$

$\leq\left(\frac{\lambda^{v-1} \lambda^{2(v-1)} \lambda^{5 v-4}}{(2 v-1)(4 v-3)(10 v-7)}\right) e^{\lambda y t}\left\|\gamma_{1}-\gamma_{2}\right\|_{B, \lambda}$.

Then

$\left\|T \gamma_{1}-T \gamma_{2}\right\|_{B, \lambda} \leq\left(\frac{\lambda^{v-1} \lambda^{2(v-1)} \lambda^{5 v-4}}{(2 v-1)(4 v-3)(10 v-7)}\right)\left\|\gamma_{1}-\gamma_{2}\right\|_{B, \lambda}$. 


\section{Al-Nahrain Journal of Science}

ANJS, Vol.23 (2), June, 2020, pp. $44-51$

So, by equation (13) $T$ is a $k$-contraction with

$$
k=\left(\frac{\lambda^{v-1} \lambda^{2(v-1)} \lambda^{5 v-4}}{(2 v-1)(4 v-3)(10 v-7)}\right)<1 .
$$

Therefore, by Banach fixed point theorem there exist a unique fixed point which represent the solution.

\section{The Approach:}

In this section, we will apply a modified semi analytic iterative method for solving problem (1)-(2). To start the approach let us present the following steps:

Step 1: Suppose that $\gamma_{0}(y, t)$ is an initial guess to problem (1)-(2) and it satisfy the following equation

$$
T_{y}^{v} \gamma_{0}(y, t)=g(y, t)
$$

subject to

$$
\gamma_{0}(0, t)=b(t)
$$

Therefore,

$$
\gamma_{0}(y, t)=b(t)+I_{y}^{v} g(y, t) .
$$

The initial guess $\gamma_{0}(y, t)$ can be decomposed by using SLPs as:

$$
\gamma_{0}^{*}(y, t)=\sum_{i=0}^{n} \sum_{j=0}^{m} c_{i j} \mathcal{L}_{1, i}(y) \mathcal{L}_{1, j}(t),
$$

where $n, m \in \mathbb{N}$ and

$$
\begin{gathered}
c_{i j}=(2 i+1)(2 j+1) \int_{0}^{1} \int_{0}^{1} \gamma_{0}(y, t) \mathcal{L}_{1, i}(y) \mathcal{L}_{1, j}(t) d y d t \\
i=0,1, \ldots, n, j=0,1, \ldots, m .
\end{gathered}
$$

Step 2: To find $\gamma_{1}(y, t)$ in the next iteration, the following initial value problem need to be solved: subject to

$$
T_{y}^{v} \gamma_{1}(y, t)=g(y, t)+I_{t}^{v} I_{y}^{v} \gamma_{0}^{*}(y, t)
$$

$$
\gamma_{1}(0, t)=b(t)
$$

Hence

$$
\gamma_{1}(y, t)=b(t)+I_{y}^{v} g(y, t)+I_{y}^{v} I_{t}^{v} I_{y}^{v} \gamma_{0}^{*}(y, s) .
$$

Similarly, $\gamma_{1}(y, t)$ also can be decomposed using SLPs as:

$$
\gamma_{1}^{*}(y, t)=\sum_{i=0}^{n} \sum_{j=0}^{m} c_{i j} \mathcal{L}_{1, i}(y) \mathcal{L}_{1, j}(t),
$$

where

$c_{i j}=(2 i+1)(2 j+1) \int_{0}^{1} \int_{0}^{1} \gamma_{1}(y, t) \mathcal{L}_{1, i}(y) \mathcal{L}_{1, j}(t) d y d t$

$i=0,1, \ldots, n, j=0,1, \ldots, m$
Step 3: For finding $\gamma_{2}(y, t), \gamma_{3}(y, t), \ldots$ etc an iterative procedure can be constructed as:

$$
T_{y}^{v} \gamma_{n+1}(y, t)=g(y, t)+I_{t}^{v} I_{y}^{v} \gamma_{n}^{*}(y, t),
$$

Subject to $\quad \gamma_{n+1}(0, t)=b(t), \quad n=1,2,3, \ldots$

i.e. $\gamma_{n+1}(y, t)=b(t)+I_{y}^{v} g(y, t)+I_{y}^{v} I_{t}^{v} I_{y}^{v} \gamma_{n}^{*}(y, t)$.

Where $\gamma_{n}^{*}(y, t)=\sum_{i=0}^{n} \sum_{j=0}^{m} c_{i j} \mathcal{L}_{1, i}(y) \mathcal{L}_{1, j}(t)$,

and

$$
\begin{gathered}
c_{i j}=(2 i+1)(2 j+1) \int_{0}^{1} \int_{0}^{1} \gamma_{n}(y, t) \mathcal{L}_{1, i}(y) \mathcal{L}_{1, j}(t) d y d t, \\
i=0,1, \ldots, n, j=0,1, \ldots, m
\end{gathered}
$$

Note that each $\gamma_{n}(y, t), n=0,1,2, \ldots$, are considered to be the solutions of problem (1)-(2).

\section{Illustrative Examples:}

To demonstrate the efficiency and accuracy of the proposed method some illustrative examples are given in this section.

Example (1): Consider the following linear CFPIDE.

$T_{y}^{0.65} \gamma(y, t)=g(y, t)+I_{t}^{0.65} I_{y}^{0.65} \gamma(y, t)$

subject to

$$
\gamma(0, t)=0
$$

$$
g(y, t)=0.5 y^{\frac{27}{20}}+0.5 t^{3} y^{\frac{27}{20}}-0.14514 t^{\frac{13}{20}} y^{\frac{53}{20}}-
$$
$0.02584 t \overline{20} y^{20}$.

The exact solution of problem (14)-(15) is $\gamma(y, t)=$ $\frac{y^{2}}{4}\left(t^{3}+1\right)$.

Table 1 represent the absolute error of problem (14)(15) for $\gamma_{3}$ and $\gamma_{5}$. Following figure 1 and figure 2 which shows a comparison between the solution resulted from the proposed method and the exact solution of problem (14)-(15) and the absolute error for $k=3,5$, respectively. 


\section{Al-Nahrain Journal of Science}

ANJS, Vol.23 (2), June, 2020, pp. $44-51$

Table 1. The absolute errors of the solutions for problem (14)-(15).

\begin{tabular}{ccc}
\hline$y$ & $\gamma_{3}(y, y)$ & $\gamma_{5}(y, y)$ \\
\hline 0 & 0 & 0 \\
0.1 & $7.117 \mathrm{e}-11$ & $1.833 \mathrm{e}-15$ \\
0.2 & $1.082 \mathrm{e}-9$ & $9.468 \mathrm{e}-15$ \\
0.3 & $4.827 \mathrm{e}-9$ & $1.794 \mathrm{e}-13$ \\
0.4 & $1.461 \mathrm{e}-8$ & $1.214 \mathrm{e}-12$ \\
0.5 & $3.622 \mathrm{e}-8$ & $5.535 \mathrm{e}-12$ \\
0.6 & $7.811 \mathrm{e}-8$ & $1.975 \mathrm{e}-11$ \\
0.7 & $1.497 \mathrm{e}-7$ & $5.92 \mathrm{e}-11$ \\
0.8 & $2.564 \mathrm{e}-7$ & $1.557 \mathrm{e}-10$ \\
0.9 & $3.912 \mathrm{e}-7$ & $3.693 \mathrm{e}-10$ \\
1 & $5.2 \mathrm{e}-7$ & $8.065 \mathrm{e}-10$ \\
\hline
\end{tabular}
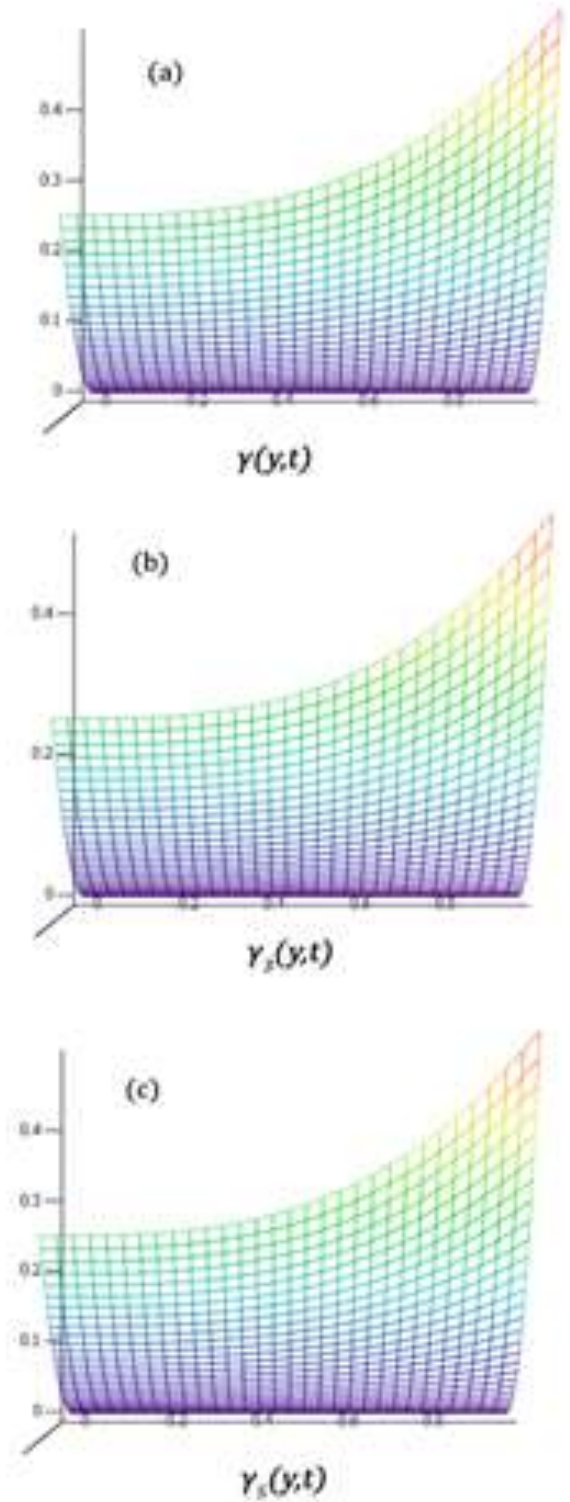

Figure 1. The compression between the proposed method and the exact solution of problem (14)-(15). (a)

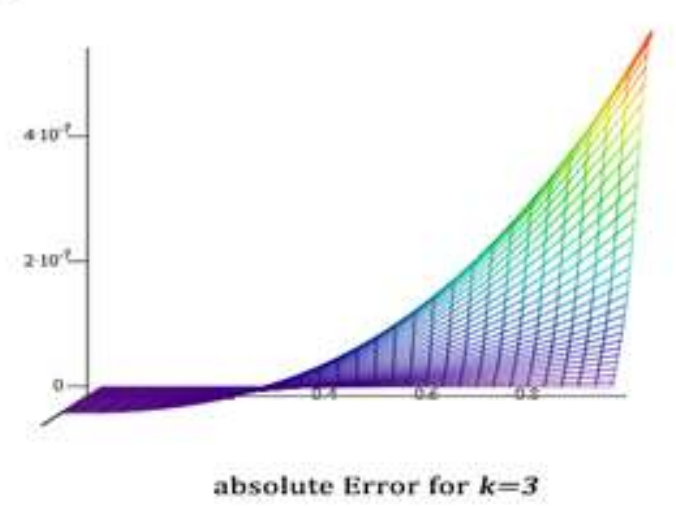

(b)

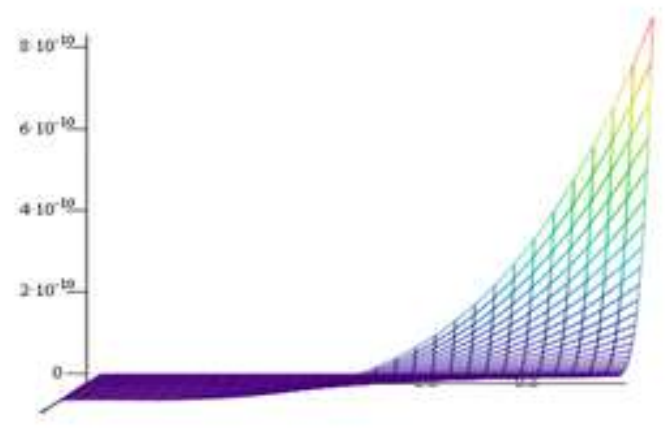

absolute error for $k=5$

Figure 2. The absolute error between the approximate solution and the exact solution of problem (14)-(15).

Example (2): Consider the following linear CFPIDEs.

$T_{y}^{0.58} \gamma(y, t)=g(y, t)+I_{t}^{0.58} I_{y}^{0.58} \gamma(y, t)$,

subject to the initial condition

$$
\begin{gathered}
\gamma(0, t)=2 t, \\
g(y, t)=2 y^{\frac{71}{50}}-2.18245 t^{\frac{79}{50}} y^{\frac{29}{50}}-0.6683 t^{\frac{29}{50}} y^{\frac{129}{50}} .
\end{gathered}
$$

The exact solution of problem (12)-(13) is $\gamma(y, t)=$ $2 t+y^{2}$.

Table 2 represent the absolute error of problem (16)(17) for $\gamma_{3}$ and $\gamma_{5}$. Following figure 3 and figure 4 which shows a comparison between the solution resulted from the proposed method and the exact solution of problem (16)-(17) and the absolute error for $k=3,5$, respectively. 


\section{Al-Nahrain Journal of Science}

ANJS, Vol.23 (2), June, 2020, pp. $44-51$

Table 2. The absolute errors of the solutions for

\begin{tabular}{ccc}
\multicolumn{3}{c}{ problem (16)- $(17)}$. \\
\hline $\boldsymbol{y}$ & $\gamma_{3}(\boldsymbol{y}, \boldsymbol{y})$ & $\gamma_{5}(\boldsymbol{y}, \boldsymbol{y})$ \\
\hline 0 & 0 & 0 \\
0.1 & $5.073 \mathrm{e}-7$ & $2.478 \mathrm{e}-9$ \\
0.2 & $9.511 \mathrm{e}-7$ & $8.222 \mathrm{e}-9$ \\
0.3 & $1.832 \mathrm{e}-6$ & $1.803 \mathrm{e}-8$ \\
0.4 & $4.58 \mathrm{e}-6$ & $3.476 \mathrm{e}-8$ \\
0.5 & $1.127 \mathrm{e}-5$ & $6.391 \mathrm{e}-8$ \\
0.6 & $2.518 \mathrm{e}-5$ & $1.154 \mathrm{e}-7$ \\
0.7 & $5.183 \mathrm{e}-5$ & $2.059 \mathrm{e}-7$ \\
0.8 & $1.006 \mathrm{e}-4$ & $3.62 \mathrm{e}-7$ \\
0.9 & $1.866 \mathrm{e}-4$ & $6.234 \mathrm{e}-7$ \\
1 & $3.332 \mathrm{e}-4$ & $1.048 \mathrm{e}-6$ \\
\hline
\end{tabular}

Example (3): Consider the following linear CFPIDEs.

$T_{y}^{0.85} \gamma(y, t)=g(y, t)+I_{t}^{0.85} I_{y}^{0.85} \gamma(y, t)$,

subject to the initial condition

$g(y, t)=$ $\gamma(0, t)=t^{3}$

$t^{2} y^{\frac{3}{20}}-3 y^{\frac{43}{20}}-2 t y^{\frac{23}{20}}+\quad 0.305577 t^{\frac{17}{20}}+y^{\frac{77}{20}}-$

$0.30557777^{\frac{77}{20}} y^{\frac{17}{20}}+0.189663 t^{\frac{37}{20}} y^{\frac{57}{20}}-$

$0.189663 t^{\frac{57}{20}} y^{\frac{37}{20}}$.

The exact solution of problem (18)-(19) is $\gamma(y, t)=$ $t^{3}+y t^{2}-y^{2} t-y^{3}$.

Table 3 represent the absolute error of problem (18)(19) for $\gamma_{3}$ and $\gamma_{5}$. Following figure 5 and figure 6 which shows a comparison between the solution resulted from the proposed method and the exact solution of problem (18)-(19) and the absolute error for $k=3,5$, respectively.

Table 3. The absolute errors of the solutions for

\begin{tabular}{ccc}
\multicolumn{3}{c}{ problem (18)- $(19)}$. \\
\hline$y$ & $\gamma_{3}(y, y)$ & $\gamma_{5}(y, y)$ \\
\hline 0 & 0 & 0 \\
0.1 & $4.653 \mathrm{e}-11$ & $3.592 \mathrm{e}-15$ \\
0.2 & $6.114 \mathrm{e}-11$ & $9.243 \mathrm{e}-15$ \\
0.3 & $1.28 \mathrm{e}-10$ & $1.798 \mathrm{e}-14$ \\
0.4 & $5.017 \mathrm{e}-10$ & $4.4 \mathrm{e}-14$ \\
0.5 & $1.703 \mathrm{e}-9$ & $1.227 \mathrm{e}-13$ \\
0.6 & $5.003 \mathrm{e}-9$ & $3.433 \mathrm{e}-13$ \\
0.7 & $1.324 \mathrm{e}-8$ & $9.301 \mathrm{e}-13$ \\
0.8 & $3.365 \mathrm{e}-8$ & $2.466 \mathrm{e}-12$ \\
0.9 & $8.73 \mathrm{e}-8$ & $0.403 \mathrm{e}-12$ \\
1 & $2.336 \mathrm{e}-7$ & $1.661 \mathrm{e}-11$ \\
\hline
\end{tabular}
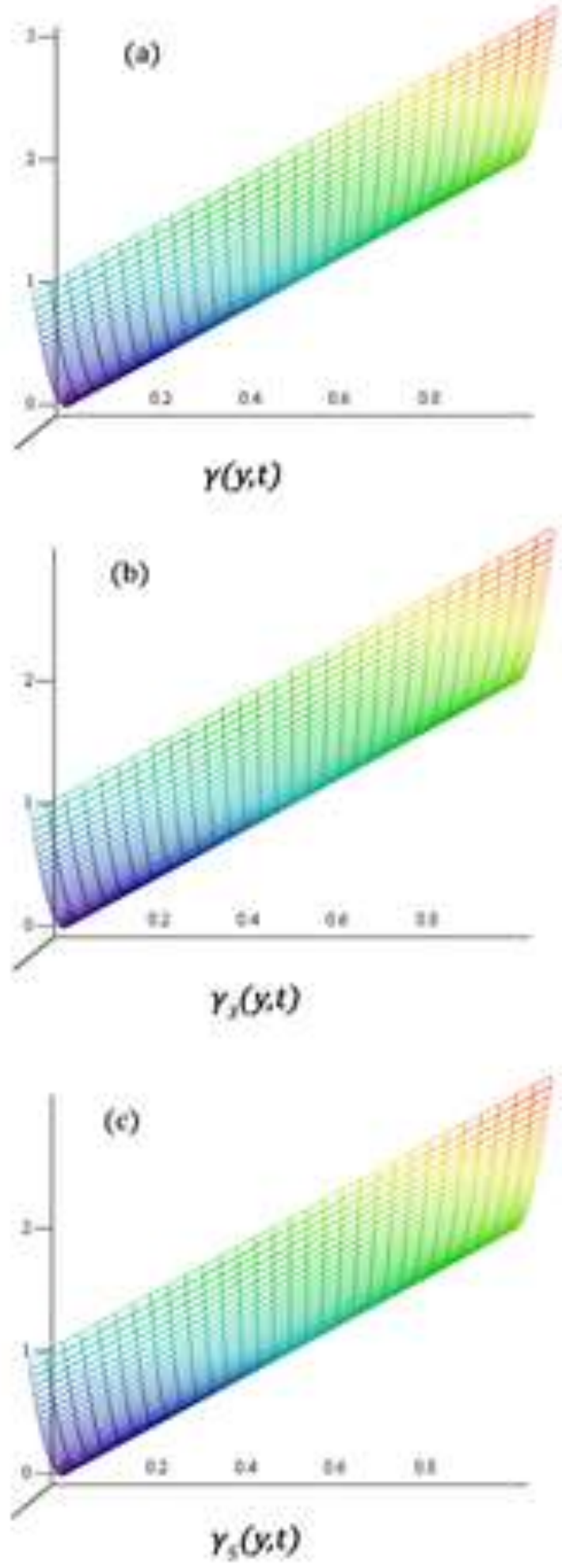

Figure 3. The compression between the proposed method and the exact solution of problem (16)-(17). 


\section{Al-Nahrain Journal of Science}

ANJS, Vol.23 (2), June, 2020, pp. $44-51$

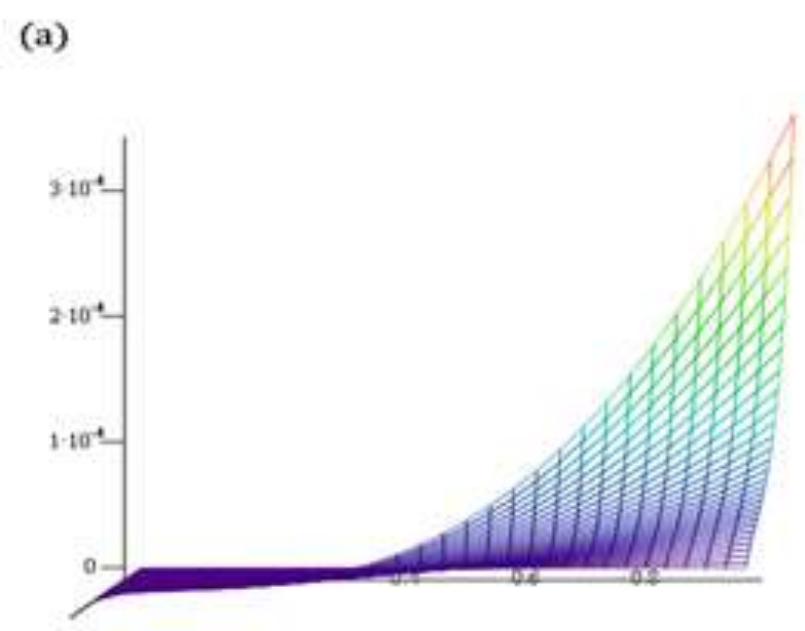

absolute Error for $\boldsymbol{k}=3$

(b)

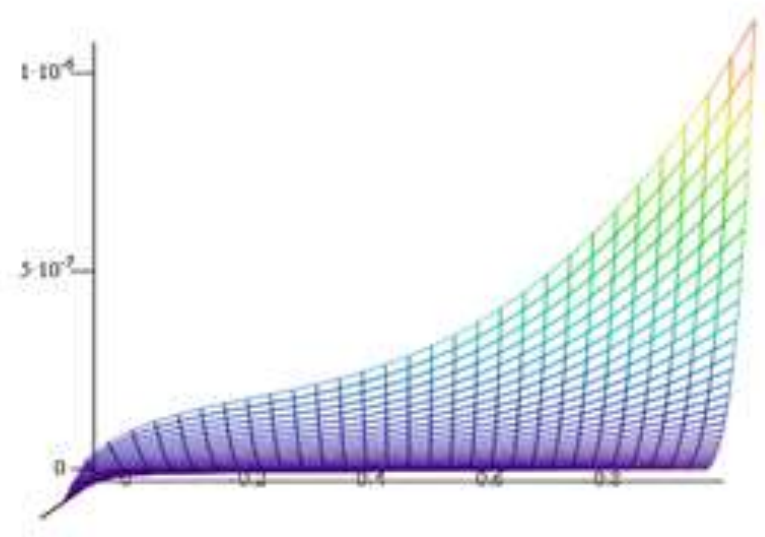

absolute error for $k=5$

Figure 4. The absolute error between the approximate solution and the exact solution of problem (16)-(17).
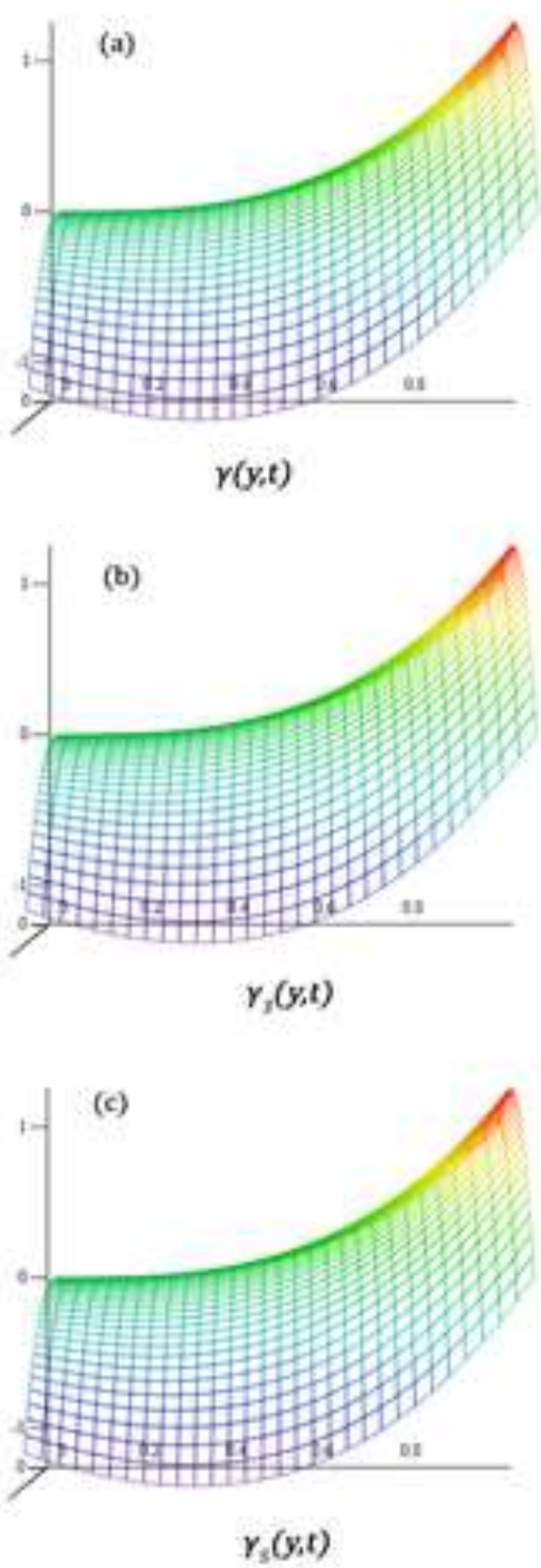

Figure 5. The compression between the proposed method and the exact solution of problem (18)-(19). 


\section{Al-Nahrain Journal of Science}

ANJS, Vol.23 (2), June, 2020, pp. $44-51$

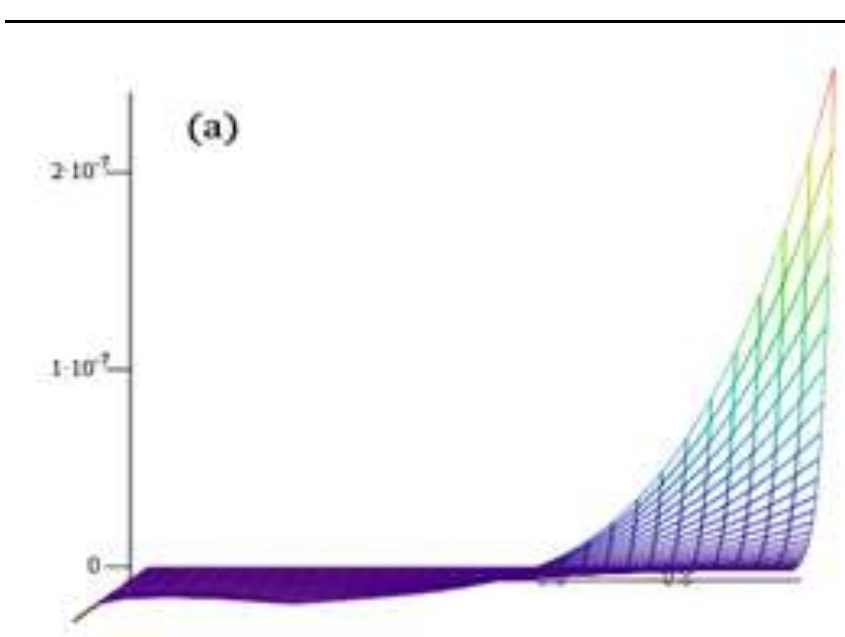

absolute Error for $k=3$

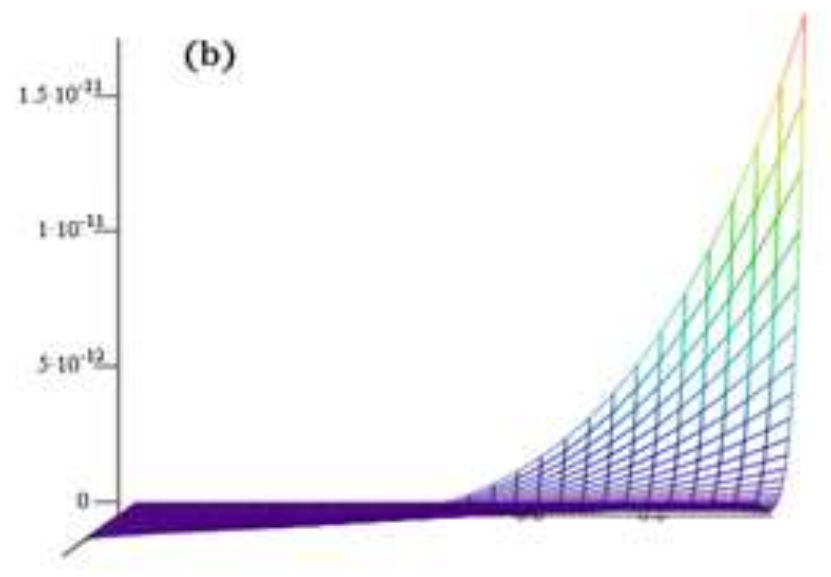

absolute error for $k=5$

Figure 6. The absolute error between the approximate solution and the exact solution of problem (18)-(19).

\section{Conclusions}

In this paper we proposed a modified semi-analytic iterative method so that one may find the solution of class of CFPIDEs. And as demonstrated in the examples, it is very effective and efficient in solving problem (1)(2). The suggested method is simple to apply and to simplify the calculations involved, the use of the shifted Legendre polynomials came in handy even without the use of a large number of polynomials.

\section{References}

[1] Oldham, K.; Spanier, J.; "The fractional calculus theory and applications of differentiation and integration to arbitrary order"; Academic Press, New York, 1974.

[2] Podlubny, I.; "Fractional Differential Equations"; Academic Press, 1999.
[3] Atangana, A.; Secer, A.; "A Note on Fractional Order Derivatives and Table of Fractional Derivatives of Some Special Functions. Abstract and Applied Analysis"; 1-8, 2013.

[4] Khalil, R.; Al Horani, M.; Yousef, A.; Sababheh, M.; "A new definition of fractional derivative, Computational and Applied Mathematics"; 65-70, 2014.

[5] Mohammed, D. S.; "Numerical solution of fractional integrodifferential equations by least squares method and shifted Chebyshev polynomial"; Math. Problems Eng. 5, 2014.

[6] El-Ajou, A.; Abu-Arqub, O.; Momani, S.; Baleanu, D.; Alsaedi, A.; "A novel expansion iterative method for solving linear partial differential equations of fractional order"; Appl. Math. Comput. 257, 119133, 2015.

[7] Rostami, Y.; Maleknejad, K.; "Numerical solution of partial integro-differential equations by using projection method"; Mediter. J. Math. 14, 2017.

[8] Akilandeeswari, A.; Balachandran, K.; Rivero, M.; Trujillo, J. J. "On the solutions of partial integrodifferential equations of fractional order"; 2017.

[9] Hamoud, A. A.; Ghadle, K. P.; "the approximate solutions of fractional volterra-fredholm integrodifferential equations by using analytical Techniques"; Probl. Anal. Issues Anal. 7 (25), 41$58,2018$.

[10] Atangana, A.; Araz, S.; "Analysis of a new partial integro-differential equation with mixed fractional operators"; Chaos, Solitons and Fractals 127, 257$271,2019$.

[11] Hendi, F. A.; Al-Qarni, M. M.; "the variational Adomian decomposition method for solving nonlinear two- dimensional Volterra-Fredholm integro-differential equation" J. King Saud Univ. 31, 110-113, 2019.

[12] Ayşegül Daşcioğlu and Dilek Varol Bayram, "Solving Fractional Fredholm Integro-Differential Equations by Laguerre Polynomials"; Sains Malaysiana 48(1), 251-257, 2019.

[13] Abdeljawad, T.; "On conformable fractional calculus"; J. Comp. Appl. Math. 279, 57-66, 2015.

[14] Bhrawy, A. H.; Doha, E. H.; Ezz-Eldien, S. S.; Abdelkawy, M. A.; "A numerical technique based on the shifted Legendre polynomials for solving the time-fractional coupled KdV equation"; SpringerVerlag Italia, 2015.

[15] Silva, F. S.; "Conformable Fractional Integral Equations of the Second Kind, Mathematica Aeterna"; vol. 8, 199-205, 2, 2018. 